\title{
Role of PSOFA-E Score in Predicting the Clinical Outcome of Critically III Children
}

\author{
Bhaskar Gorla', Vishwas Rao² \\ ${ }^{1}$ Junior resident, Department of Pediatrics , AJ Institute of Medical Sciences , Kuntikana , Mangalore, ${ }^{2}$ Senior resident, Department of Pediatrics , AJ Institute of Medical \\ Sciences, Kuntikana, Mangalore.
}

\section{Abstract}

Background: Recently pediatric sequential organ failure assessment (pSOFA) score was adapted and validated in critically ill children. This study was aimed to evaluate the feasibility of addition of echocardiographic parameters to paediatric version of SOFA score (pSOFA-E score) and to adapt and validate with reference to pSOFA score in predicting the mortality of critically ill children. Subjects and Methods: This hospital based prospective, observational, analytical study was conducted in the Department of Paediatrics, A. J Hospital, Mangalore, Karnataka, from November 2017 to November 2019. A total of 74 cases were studied. Result: Most of the children were aged <1 year (41.89\%). Majority of the patients (62.16\%) improved and $37.84 \%$ of the patients expired. The mean and median pSOFA-E scores were $10.53 \pm 4.06$ and 10 respectively and pSOFA-E score of 5-8 was noted in most of the children (32.43\%). Mortality was significantly high in children with pSOFA-E score between 9-12 (39.13\%), 13-16 (77.78\%), 17-20 (83.33\%) (p<0.001). Receiver operating characteristic curve (ROC) yielded area under curve (AUC) of 0.920 and 0.791 with a cut-off value of 11.5 in predicting mortality. Significantly higher number of children with pSOFA-E score of $\geq 11.5$ had positive blood culture $(30 \%)$. Conclusion: The findings of the present study validate and emphasize that, addition of score devised by requirement of ionotropes to maintain adequate ejection fraction defy simple bedside echocardiography to pSOFA score is highly useful and accurate in discrimination of PICU mortality, morbidity and cardiovascular status/ compromise of body.

Keywords: Critical illness; Ejection fraction; Pediatric sequential organ failure assessment.

Corresponding Author: Dr. Vishwas Rao, Senior Resident, Department of Pediatrics, AJ Institute of Medical Sciences, Kuntikana, Mangalore, A- 1603, Brigade Pinnacle, Derebail, Mangalore.

Email: bhaskargoarla@gmail.com

Received: January 2020

Accepted: January 2020

\section{Introduction}

Several MODS scoring systems have been developed and validated mainly in adults.However, an equivalent MODS scoring system is not available for critically ill children. The ideal probability model / scoring system would be institution independent and population independent. The Sequential Organ Failure Assessment (SOFA) score was selected as the scoring system to quantify organ dysfunction in the Third International Consensus Definitions for Sepsis and Septic Shock (Sepsis-3).TheSepsis-3Task Force validated the SOFA score in adult patients with suspected infection and found the SOFA system to be either comparable or superior to other scoring systems at discriminating in-hospital mortality. The Sepsis-3 definitions are expected to be widely adopted and, by extension, the use of SOFA score in patients with confirmed or suspected infection. ${ }^{[1]}$

Echocardiography is currently considered a key tool for the hemodynamic assessment in Intensive Care Units (ICU), able to identify causes of hemodynamic instability and to quickly guide therapy.Some of its advantages are being a noninvasive method, risk-free, capable of being performed serially and in real time, and analyzed along with clinical data by intensivists. ${ }^{[2]}$ Several studies have demonstrated the positive effect of the use of echocardiography in the management of critically ill patients, changing their treatment in $30 \%-60 \%$ of cases after the test is performed. ${ }^{[3]}$ Scoring systems are arrived at evaluation of the patient's mortality risk in the ICU by assigning a score to patient and predicting the outcome. ${ }^{[4,5,6]}$ Therefore there is need for effective scoring system from the one in which they were originally developed. However, till date none of the studies have focused on the relationship between SOFA score and echocardiography. Although, echocardiography a key tool for the hemodynamic assessment along with SOFA as an organ dysfunction scale have different functions, an association between the two might provide some clinical implications which may be strong predictor of outcomes among critically ill children ${ }^{[2]}$ Hence we sought to determine applicability of SOFA-Echocardiography (ECHO) [pSOFAE] score in predicting the mortality of critically ill children.

Subjects and Methods 
This was a hospital based prospective, observational, analytical study conducted in PICU of Tertiary care hospital over a period of 2 years. Children admitted to PICU with paediatric MODS were enrolled. During the study period there were 312 number of admissions. Of them, 75 children were eligible for the study. One case was discharged against medical advice for which the outcome was not available. Hence a total of 74 cases were studied after obtaining a written informed consent from caregivers. Prior to commencement, ethical clearance was obtained from Ethical and Research Committee, A J Institute of Medical Sciences, Kuntikana, Mangalore, Karnataka.

\section{Inclusion Criteria}

- Children admitted to PICU with paediatric MODS (more than one organ system failure) irrespective of the cause.

- Children aged between one month to 18 years.

\section{Exclusion criteria}

- Children admitted to PICU for scheduled procedures normally cared for in a PICU (like hemodialysis, Intravenous Immunoglobulin [IVIG] administration).

- Children with PICU stay of $<24$ hours.

A thorough clinical and systemic examination was conducted and Glassgow coma score (GCS) score were calculated. These findings were recorded on a predesigned and pretested proforma.

Under aseptic precautions, blood samples of $5 \mathrm{~mL}$ were collected to determine the Total count, Platelet count, Total bilirubin, Serum Creatinine, C Reactive Protein, Blood culture, Arterial Blood gas analysis to analyse the pSofa Score.

In the present study in an attempt to improve the diagnostic performance we evaluated the hemodynamic condition of the patient based on transthoracic echocardiography (TTE) at bedside by the pediatric cardiologist. The ejection fraction was considered for the evaluation which was graded as below and this score was added to the pSOFA score devised by Mattics TJ and Sanchez-Pinto LN. ${ }^{20}$ in order to calculate pSOFA-E score.

Grading of Ejection fraction ${ }^{7}$

- Score of $0=$ Ejection fraction of $55 \%$

- Score of 1 = Ejection fraction of $55 \%$ with one ionotrope

- Score of 2 = Ejection fraction of 55\% with more than one ionotrope.

The data obtained was coded and entered into Microsoft excel spreadsheet. Categorical data was expressed as rates, ratios and percentages. Continuous data was expressed as mean \pm standard deviation. The comparison of categorical data was done by chi-square test and/or Fishers exact test and independent sample ' $t$ ' test was used to compare mean values. The discrimination of pSOFA and pSOFA-E score in the diagnosis of sepsis and survival was done using was made by area under the curve (AUC) using the receiver operating characteristic curve (ROC curve).$^{96}$ If the AUC (Area under curve) is 0.9 or more its considered excellent discrimination, $0.80-0.89$ it considered good and 0.70-0.79 as fair. The accuracy of pSOFA and pSOFA-E score in discriminating the survival was expressed in terms of sensitivity, specificity, positive predictive value (PPV), negative predictive value (NPV) and positive likelihood ratio (PLR) and negative likelihood ratio (NLR). At 95\% confidence interval, a probability (p) value of $\leq 0.050$ was considered as statistically significant. At $95 \%$ confidence interval (CI), a probability value of less than or equal to 0.05 was considered as statistically significant.

\section{Results}

The data obtained was tabulated and analysed. The final results were tabulated and interpreted as below.

Table 1: Clinical profile of the study population

\begin{tabular}{|l|l|l|l|l|l|}
\hline \multirow{2}{*}{ Parameters } & \multicolumn{3}{|l|}{ Mean $(\mathrm{n}=74)$} & Median & Range \\
\cline { 2 - 3 } \cline { 5 - 6 } & Mean & $\mathrm{SD}$ & & Minimum & Maximum \\
\hline Age (Years) & 4.34 & 5.46 & 1.00 & 0.08 & 16.00 \\
\hline $\begin{array}{l}\text { Total count }(\mathrm{x} \\
1000 \text { per cumm) }\end{array}$ & 12.31 & 5.44 & 11.80 & 3.70 & 33.60 \\
\hline $\begin{array}{l}\text { Platelet count }(\mathrm{x} \\
\text { lakhs per cumm) }\end{array}$ & 2.21 & 1.47 & 1.95 & 0.16 & 6.35 \\
\hline $\begin{array}{l}\text { Total bilirubin } \\
\text { (mg/dL) }\end{array}$ & 1.03 & 1.22 & 0.70 & 0.20 & 7.90 \\
\hline $\begin{array}{l}\text { Ionotrope dose } \\
\text { (mcg/Kg/min) }\end{array}$ & 10.73 & 2.44 & 10.00 & 5.00 & 15.00 \\
\hline $\begin{array}{l}\text { Mean arterial } \\
\text { pressure (mm } \\
\text { Hg) }\end{array}$ & 50.16 & 13.34 & 48.50 & 28.00 & 88.00 \\
\hline $\begin{array}{l}\text { Serum creatinine } \\
\text { (mg/dL) }\end{array}$ & 0.73 & 0.52 & 0.60 & 0.30 & 3.80 \\
\hline FiO & 0.54 & 0.28 & 0.50 & 0.21 & 1.00 \\
\hline GCS & 8.85 & 3.16 & 9.00 & 3.00 & 15.00 \\
\hline pSOFA score & 9.72 & 3.51 & 10.00 & 3.00 & 17.00 \\
\hline pSOFA-E Score & 10.53 & 4.06 & 10.00 & 3.00 & 19.00 \\
\hline $\begin{array}{l}\text { Length of } \\
\text { hospital stay } \\
\text { (Days) }\end{array}$ & 11.00 & 8.60 & 8.00 & 3.00 & 43.00 \\
\hline
\end{tabular}

The clinical characteristics of the study population are as shown in table The age of the children ranged between 0.08 to 16 years. The mean and median age was noted as $12.31 \pm 5.44$ years and 11.80 years. It was observed that the mean and median PSOFA score was $9.72 \pm 0.51$ and 10 respective and it ranged between 3 to 17 . The mean and median pSOFA-E score was $10.53 \pm 4.06$ and 10 respective and it ranged between 3 to 19 .

In the present study $62.16 \%(\mathrm{n}=46)$ of the patient improved and $37.84 \%(n=28)$ of the patients expired. 


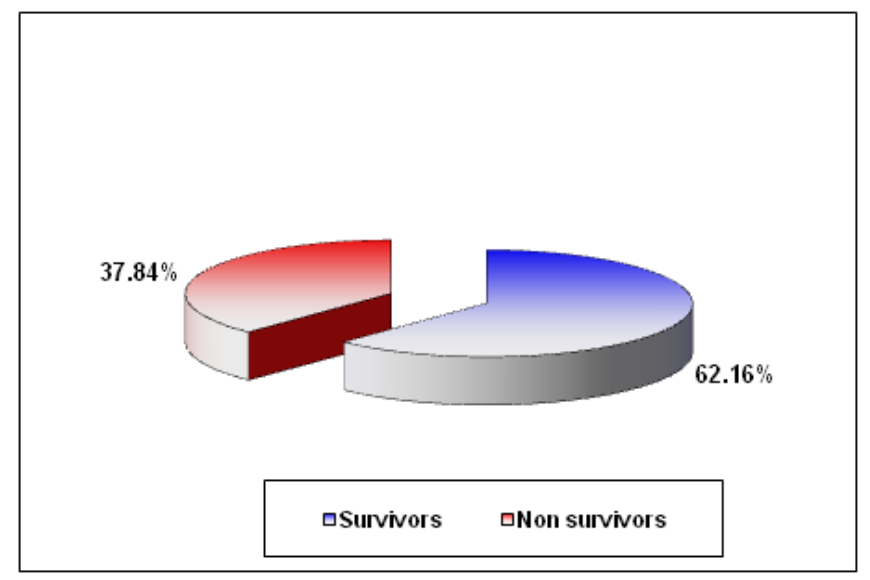

Graph 1: Clinical profile of the study population

Table 2: Distribution of children according to the pSOFA-E score and its association with outcome

\begin{tabular}{|l|l|l|l|l|l|l|}
\hline \multirow{2}{*}{ pSOFA-E score } & \multicolumn{2}{l|}{ Outcome } & \multicolumn{2}{l|}{ Total } \\
\cline { 2 - 6 } & Survivors & \multicolumn{2}{l|}{ Non survivors } & \multicolumn{2}{l|}{} \\
\cline { 2 - 6 } & No. & \% & No. & \% & No. & \% \\
\hline 0 to 4 & 3 & 100.00 & 0 & 0.00 & 3 & 4.05 \\
\hline 5 to 8 & 24 & 100.00 & 0 & 0.00 & 24 & 32.43 \\
\hline 9 to 12 & 14 & 60.87 & 9 & 39.13 & 23 & 31.08 \\
\hline 13 to 16 & 4 & 22.22 & 14 & 77.78 & 18 & 24.32 \\
\hline 17 to 20 & 1 & 16.67 & 5 & 83.33 & 6 & 8.11 \\
\hline Total & 46 & 62.16 & 28 & 37.84 & 74 & 100.00 \\
\hline
\end{tabular}

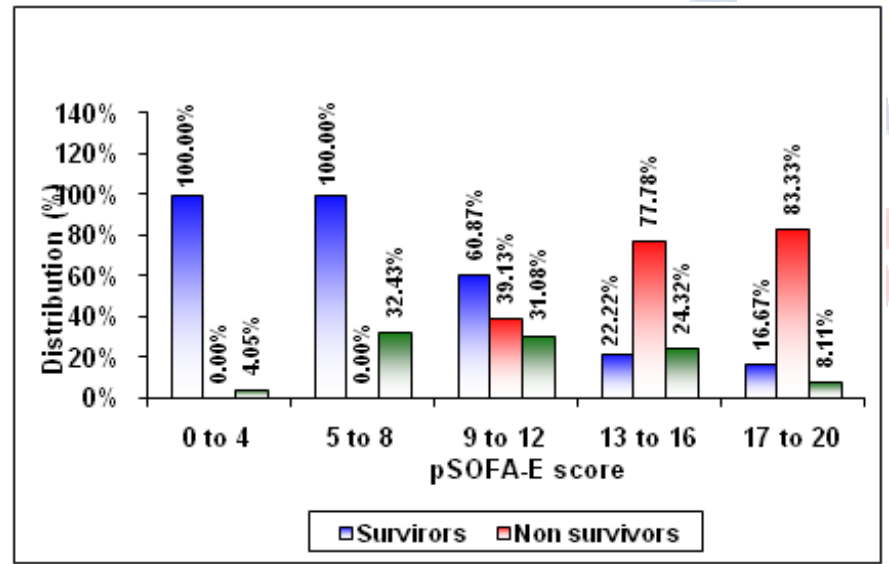

Graph 2: Clinical profile of the study population

In this study pSOFA-E score of 5 to 8 was noted in most of the children $(32.43 \%)$. Mortality was significantly high in children with pSOFA-E score between 9 to 12 (39.13\%), 13 to $16(77.78 \%), 17$ to $20(83.33 \%)(\mathrm{p}<0.001)$.

Table 3: Distribution of children according to the ejection fraction and its association with outcome

\begin{tabular}{|l|l|l|l|l|l|l|}
\hline \multirow{2}{*}{$\begin{array}{l}\text { Ejection fraction } \\
\text { score }\end{array}$} & \multicolumn{2}{|l|}{ Outcome } & \multicolumn{2}{l|}{ Total } \\
\cline { 2 - 7 } & \multicolumn{2}{|l|}{ Survivors } & \multicolumn{2}{|l|}{ Non survivors } & \multicolumn{2}{l|}{} \\
\cline { 2 - 7 } & No. & $\%$ & No. & $\%$ & No. & $\%$ \\
\hline $0(>55 \%)$ & 30 & 90.91 & 3 & 9.09 & 33 & 44.59 \\
\hline $\begin{array}{l}1(55 \% \text { with } 1 \\
\text { ionotrope) }\end{array}$ & 12 & 54.55 & 10 & 45.45 & 22 & 29.73 \\
\hline $\begin{array}{l}2(55 \% \text { with }>1 \\
\text { ionotrope) }\end{array}$ & 4 & 21.05 & 15 & 78.95 & 19 & 25.68 \\
\hline Total & 46 & 62.16 & 28 & 37.84 & 74 & 100.00 \\
\hline
\end{tabular}

In this study echocardiography score of zero was noted in in most of the children (44.59\%). Mortality was significantly high in children with echocardiography score of two $(78.95 \%)$ followed by one $(45.45 \%)(\mathrm{p}<0.001)$.

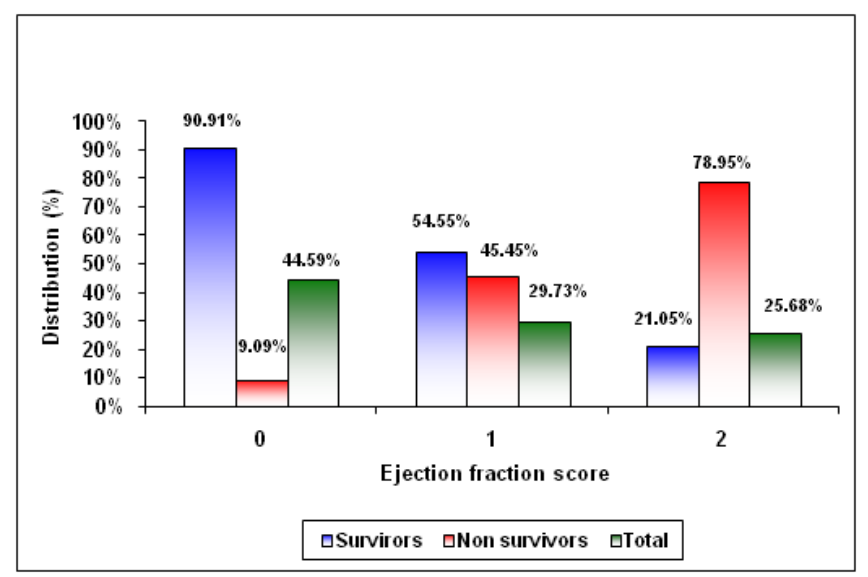

Graph 3: Distribution of children according to the ejection fraction and its association with outcome

In this study echocardiography score of zero was noted in in most of the children (44.59\%). Mortality was significantly high in children with echocardiography score of two $(78.95 \%)$ followed by one $(45.45 \%)(\mathrm{p}<0.001)$.

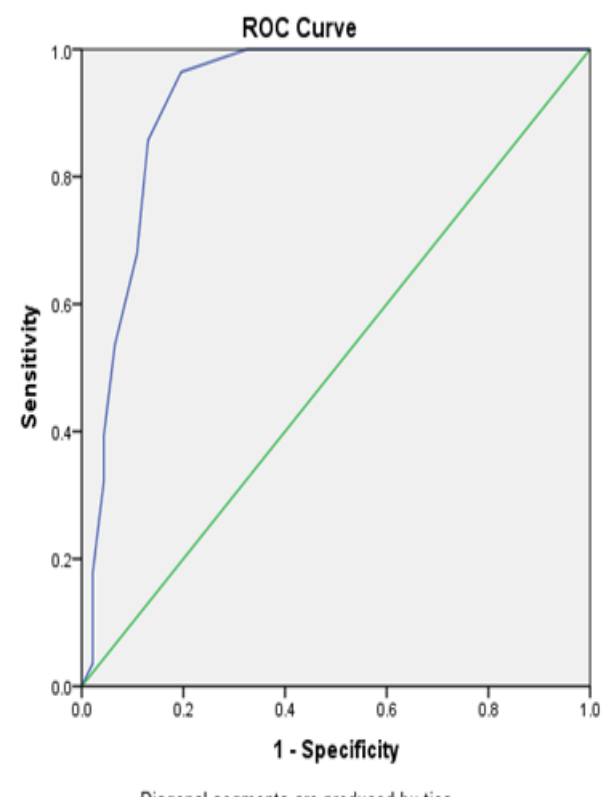

Diagonal segments are produced by ties.

Graph 4: Receiver operating characteristic curve in predicting mortality for pSOFA-E score

AUC $=0.920$; Std error $=0.032 ; 95 \% \mathrm{CI}=0.857$ to $0.984 ; \mathrm{p}<0.001$; Cut off value $=11.5$ for $\mathrm{pSOFA}-\mathrm{E}$

In the present study the ROC yielded AUC of 0.920 (Std error $=0.032 ; 95 \% \mathrm{CI}=0.857$ to $0.984 ; \mathrm{p}<0.001$ ) and a cut-off value of 11.5 in predicting mortality for pSOFA-E score. 


\section{Garla \& Raa; Predicting the Clinical Outcame of Critically IUl Children}

Table 4: Association of pSOFA-E score with outcome and its accuracy in predicting outcome

\begin{tabular}{|l|l|l|l|l|l|l|}
\hline \multirow{2}{*}{ pSOFA-E score } & \multicolumn{2}{|l|}{ Outcome } & \multicolumn{2}{l|}{ Total } \\
\cline { 2 - 6 } & Survivors & \multicolumn{2}{l|}{ Non survivors } & \multicolumn{2}{l|}{} \\
\cline { 2 - 6 } & No. & \% & No. & \% & No. & \% \\
\hline$\geq 11.5$ & 6 & 20.00 & 24 & 80.00 & 30 & 15.00 \\
\hline$<11.5$ & 40 & 90.91 & 4 & 9.09 & 44 & 22.00 \\
\hline Total & 46 & 62.16 & 28 & 37.84 & 74 & 100.00 \\
\hline
\end{tabular}

Sensitivity $=85.71 \% ; \quad$ specificity $=86.96 \% ; \quad \mathrm{PPV}=80 \% ; \quad \mathrm{NPV}=90.91 \%$; Diagnostic accuracy $=86.49 \% ;$ PLR $=6.57 ;$ NLR $=0.16 ; p<0.001$

In the present study significantly higher number of children with pSOFA-E score of $\geq 11.5$ expired $(80 \%)$. The diagnostic accuracy of pSOFA-E score in predicting outcome was $86.49 \%$ with sensitivity of $85.71 \%$, specificity of $86.96 \%$, PPV of $80 \%$ and NPV of $90.91 \%$ (PLR=6.57; $\mathrm{NLR}=0.16 ; \mathrm{p}<0.001$ ).

\section{Discussion}

The present study was planned to evaluate the feasibility of addition of echocardiographic parameters to paediatric version of SOFA score so as to formulate the pSOFAECHO [pSOFA-E] score and to adapt and validate with reference to pSOFA score in predicting the mortality of critically ill children in our settings, which is first of its kind. The present hospital based prospective, observational, analytical study was conducted in the Paediatric Intensive Care Unit, A. J Hospital, Mangalore, Karnataka, from November 2017 to November 2019. During the study period there were 312 numbers of admissions and of which, 75 children fulfilled the selection criteria and were eligible. Further out of 75 eligible cases one case was discharged against medical advice for which the outcome was not available. Hence a total of 74 cases were studied.

In the present study, though there was wide variation in the clinical presentation and diagnosis. Nearly three fourth of the patients improved $(62.16 \%)$ and more than one third (37.84\%) expired.

In this study the age of the children ranged between 0.08 to 16 years. The mean and median age was noted as $12.31 \pm 5.44$ years and 11.80 years. Most of the children were aged $<1$ year $(41.89 \%)$. Although the mortality was high in children aged between 13 to 18 years $(72.73 \%)$ and less than one year $(67.74 \%)$. no association was found between mortality and age $(\mathrm{p}=0.647)$.

In the present study ionotrope administration was noted in $74.32 \%$ of the children. Mortality was significantly high in children who underwent ionotrope administration (47.27\%; $\mathrm{p}=0.004$ ).

In this study, pSOFA score ranged from 3 to 17 . The mean and median pSOFA scores were $9.72 \pm 0.51$ and 10 respectively. pSOFA score of 9 to 12 was noted in most of the children (39.19\%). Mortality was significantly high in children with pSOFA score of 17 to 20 (50\%), 13 to 16
(50\%), 13 to $16(85.71 \%)$ and 9 to $12(51.72 \%)(\mathrm{p}<0.001)$ Also, positive blood culture was significantly high in children with pSOFA score between 13 to 16 (35.71\%) $(\mathrm{p}=0.021)$. Further, the ROC yielded AUC of 0.910 (Std error $=0.034 ; 95 \% \mathrm{CI}=0.843$ to $0.978 ; \mathrm{p}<0.001$ ) and a cut-off value of 10.5 in predicting mortality for pSOFA score. Similarly, the ROC yielded AUC of 0.772 (Std error $=0.059$; 95\% $\mathrm{CI}=0.843$ to $0.978 ; \mathrm{p}<0.001)$ and a cut-off value of 10.5 in predicting culture positive sepsis using pSOFA score. These observations were consistent with a study by Mattics TJ and Sanchez-Pinto $\mathrm{LN}^{1}$ who reported that, the maximum pSOFA score had excellent discrimination for inhospital mortality, with an AUC of 0.94 (95\%CI, 0.92-0.95). The optimal pSOFA cut off to discriminate mortality was a score higher than 8 points which was 10.5 in the present study with AUC of 0.910 .

Echocardiography is a key tool for hemodynamic assessment in Intensive Care Units (ICU) ${ }^{8}$. Focused echocardiography performed by nonspecialist physicians has a limited scope, and the most relevant parameters assessed by focused echocardiography in Pediatric ICU are left ventricular systolic function, fluid responsiveness, cardiac tamponade and pulmonary hypertension. Proper ability building of pediatric emergency care physicians and intensivists to perform focused echocardiography is feasible and provides improved care of severely ill children and thus should be encouraged ${ }^{9}$. In this study echocardiography score of zero was noted in in most of the children (44.59\%). Mortality was significantly high in children with echocardiography score of two $(78.95 \%)$ followed by one $(45.45 \%)(\mathrm{p}<0.001)$ suggesting that children with lower ejection fraction are at high risk of mortality.

Echocardiography is currently considered a key tool for the hemodynamic assessment in Intensive Care Units (ICU), able to identify causes of hemodynamic instability and to quickly guide therapy. Some of its advantages are being a noninvasive method, risk-free, capable of being performed serially and in real time, and analyzed along with clinical data by intensivists. ${ }^{2}$ Considering these facts we performed bedside echocardiography and added the score obtained to pSOFA score so as to determine a new scoring system pSOFA-E score. Accordingly, the pSOFA-E score in the present study ranged from 3 to 19 . The mean and median pSOFA-E score was $10.53 \pm 4.06$ and 10 respective and it ranged from 3 to 19 . Suggesting a difference of one point compared to mean pSOFA score (10.53 \pm 40.6 vs $9.72 \pm 3.51)$. Further, pSOFA-E score of 5 to 8 was noted in most of the children (32.43\%). Mortality was significantly high in children with pSOFA-E score between 9 to 12 (39.13\%), 13 to $16(77.78 \%), 17$ to $20(83.33 \%)(\mathrm{p}<0.001)$ suggesting a trend towards higher mortality with higher score. The ROC yielded AUC of 0.920 (Std error $=0.032 ; 95 \% \mathrm{CI}=0.857$ to $0.984 ; \mathrm{p}<0.001)$ and a cut-off value of 11.5 in predicting mortality for pSOFA-E score while, AUC of 0.791 (Std error $=0.052 ; 95 \% \mathrm{CI}=0.690$ to $0.888 ; \mathrm{p}=0.003$ ) and a cut-off 
value of 11.5 in predicting positive blood culture for pSOFA-E score. Furthermore, significantly higher number of children with pSOFA-E score of $\geq 11.5$ expired $(80 \%)$. The diagnostic accuracy of pSOFA-E score in predicting outcome was $86.49 \%$ with sensitivity of $85.71 \%$, specificity of $86.96 \%$, PPV of $80 \%$ and NPV of $90.91 \%$ (PLR=6.57; NLR=0.16; $\mathrm{p}<0.001)$. The comparison of various predictors for pSOFA and pSOFA-E are as depicted in table below.

This maiden study confirms that, addition of haemodynamic stability as assessed by simple bedside echocardiography and measured on the basis of requirement of ionotropes to maintain ejection fraction of $55 \%$ to pSOFA score is highly accurate in discrimination of PICU mortality. Hence it can be applied in the PICU settings.

\section{Strength}

The strength of the study was that, the scores were calculated from the data available at 72 hours which reflect the true state of the patient rather than admission scores.

\section{Limitations}

However, these conclusions require further validation due to potential limitations of this study. The conclusions drawn from this study were based on the data from a single center involving relatively a smaller sample which limits it generalizability to the entire population and lack of similar data in the literature to compare. Also longterm outcome was not considered and the scores were not validated with other organ dysfunction scoring systems like PELOD, PMODS as it was beyond the scope of this study.

\section{Recommendations}

Further large multicentric studies involving large sample size with longer duration of follow up and validating with other organ dysfunction scoring systems like PELOD, PMODS may not only help in improving the accuracy of pSOFA-E score but also PICU outcomes.

\section{Conclusion}

The findings of the present study emphasize that doing a simple bedside echocardiography and using PSOFA-E score in Paediatric Intensive Care Unit will enable us to anticipate the need of inotropes to maintain adequate ejection fraction and predict PICU mortality, morbidity and cardiovascular status of the child more accurately.

Hence PSOFA-E can be applied in the PICU settings more often thus enabling the treating physician in quick assessment of outcome of the action, intervention and treatment taken up for individual cases.

PSOFA-E if routinely applied in PICU setting will further guide us in enhancing the quality of treatment modality as a standard comparative tool is anytime preferred over any other subjective parameter.

\section{References}

1. Matics TJ, Sanchez-Pinto LN. Adaptation and Validation of a Pediatric Sequential Organ Failure Assessment Score and Evaluation of the Sepsis-3 Definitions in Critically Ill Children. JAMA Pediatr 2017;171(10):e172352.

2. 2.Gaspar HA, Morhy SS. The Role of Focused Echocardiography in Pediatric Intensive Care: A Critical Appraisal. Biomed Res Int 2015; 2015:596451.

3. McLean AS. Echocardiography in shock management. Crit Care 2016;20: Article No. 275.

4. Patel S, Vasavada H, Patel P, Patel B, Shukla P. Study of PRISM III Score as A Predictor of Mortality in PICU. J Pediatr Crit Care 2019;6(3):21-4.

5. Sahoo B, Patnaik S, Mishra R, Jain MK. Morbidity pattern and outcome of children admitted to a paediatric intensive care unit of Eastern India. Int J Contemp Pediatr 2017;4:486-9.

6. Rashma RP, Remya S, Jaykumar C, Shanavas M, Manu R, Remya R, et al. Mortality Profile of Children Admitted to Intensive Care Unit of a Tertiary Care Hospital in Kerala, South India. Int J Med Clin Sci 2018; 1.1:13-6.

7. Rodriguez F. Ejection Fraction Has a U-Shaped Relationship with Mortality. Available from: URL: https://www.jwatch.org/na49746/2019/08/28/ ejection -fraction-hasu-shaped-relationship-with-mortality Access Date 22.11.2019.

8. Bhalala U, Bansal A, Chugh K. Advances in Pediatric Critical Care Research in India. Front Pediatr 2018;6:150.

9. Şahin S, Uysal Yazıcı M, Ayar G, Köksal T, Çetin İI, et al. Clinical impact and efficacy of bedside echocardiography on patient management in pediatric intensive care units (PICUs): A prospective study. Anatol J Cardiol 2017;18(2):136-141.

Copyright: (C) the author(s), 2020. It is an open-access article distributed under the terms of the Creative Commons Attribution License (CC BY 4.0), which permits authors to retain ownership of the copyright for their content, and allow anyone to download, reuse, reprint, modify, distribute and/or copy the content as long as the original authors and source are cited.

How to cite this article: Gorla B, Rao V. Role of PSOFA-E Score in Predicting the Clinical Outcome of Critically Ill Children. Asian J. Clin. Pediatr. Neonatol.2020;8(1):27-31.

DOI: dx.doi.org/10.47009/ajcpn.2020.8.1.7

Source of Support: Nil, Conflict of Interest: None declared. 